\title{
Información básica sobre recursos del Sistema Nacional de Salud, 2001
}

\section{Presentación}

E 1 Sistema Nacional de Información en Salud está constituido por diversos subsistemas que producen información de la infraestructura, producción de servicios y resultados en salud de las diferentes instituciones que componen el Sistema Nacional de Salud en México. En particular, la infraestructura se relaciona con los recursos para la salud que, generalmente, se clasifican en físicos o materiales, humanos y financieros.

Los recursos humanos son particularmente importantes porque los sistemas de salud requieren de una mano de obra intensiva y el gasto en el personal de salud suele ser el rubro individual más importante del gasto corriente de los servicios de salud. Los sistemas de salud requieren personal calificado y experimentado para funcionar bien, y de una mezcla apropiada de los diferentes tipos de recursos humanos.

La cantidad de personal de salud depende de las decisiones de rectoría del sistema de salud sobre las inversiones en educación y los incentivos diseñados para mantener al personal en el sistema, así como para alentar o desalentar la migración entre entidades federativas o internacional.

Los recursos humanos pueden definirse como el inventario de todos los individuos comprometidos en acciones de salud, ya sea en la promoción, la protección o el mejo- ramiento de la salud. Esto incluye los sectores privado y público y los diferentes dominios de los sistemas de salud tales como atención personal preventiva y curativa, intervenciones de salud pública no personales, promoción de la salud y prevención de enfermedades.

Comentarios similares pueden hacerse en el caso del capital físico (recursos materiales); en este caso, el déficit en el inventario de activos (edificios y equipo, por ejemplo) puede ser una restricción real para la entrega de intervenciones efectivas en el corto plazo. En oposición, es frecuente encontrar sistemas de salud en los que ha habido una inversión significativa en infraestructura física, pero cuyos presupuestos recurrentes no permiten un financiamiento del personal o el mantenimiento requerido para trabajar el capital, lo que desemboca en pérdidas de eficiencia. En el largo plazo, las inversiones son costos variables y los formuladores de política necesitan planear cuidadosamente las inversiones futuras para evitar la escasez o un excedente de capacidades.

Los recursos físicos o materiales se refieren al inventario de activos productivos no-humanos incluyendo hospitales, vehículos y equipo. La información sobre la inversión física es esencial para los formuladores de política, puesto que la toma de decisiones sobre la asignación de recursos remite al presente, en oposición a inversiones que apoyarían la prestación de servicios de salud en el futuro. En este caso, si bien puede ser interesante contar con un gran número de indicadores, esto plantea una considerable demanda sobre los sistemas de información de la salud, por lo que poco a poco se deberá construir un conjunto parsimonioso diseñado para proporcionar un discernimiento de los aspectos más importantes de la función de generación de recursos.

A continuación se presentan 10 cuadros con datos absolutos y relativos a la población de los recursos humanos y materiales del componente público del Sistema Nacional de Salud para el año 2001. La información se presenta por entidad federativa y en ocasiones se desagrega por población derechohabiente y no derechohabiente, llegando, incluso, al desglose por institución. En particular, es importante mencionar que en el cuadro $X$ se presenta la relación entre camas censables y no censables en dos momentos en el tiempo, 1997 y 2001, por entidad federativa. El interés de mostrar este indicador es el de seguir con más detalle la modernización de las unidades hospitalarias de las entidades federativas que incrementan el número de camas no censables (corta estancia) en relación con las camas censables.

La calidad de la información presentada es variable por institu- 
ción y entidad federativa. Aunque adolece de problemas comunes como es el caso de recursos humanos y, particularmente, lo relativo a médicos. En realidad, por la forma en que se recolecta la información, cuando se habla de médicos se refiere a plazas, ya que un médico puede prestar sus servicios en dos instituciones. Algo similar sucede en el caso de las enfermeras, aunque el problema de traslape institucional es en menor escala. Un sistema nominal es el ideal y por lo pronto se pueden utilizar los datos recogidos por el Censo de Población y Vivienda para conocer el margen de error de los sistemas de registro.

\section{Cuadro I \\ Principales recursos físicos y materiales por entidad federativa. Sistema Nacional de Salud. México, 2001*}

\begin{tabular}{|c|c|c|c|c|c|c|c|c|c|c|}
\hline \multirow[b]{2}{*}{ Entidad federativa } & \multicolumn{3}{|c|}{ Unidades de salud } & \multicolumn{2}{|c|}{ Camas } & \multirow[b]{2}{*}{ Consultorios } & \multirow[b]{2}{*}{ Quirófanos } & \multirow[b]{2}{*}{$\begin{array}{l}\text { Salas de } \\
\text { expulsión }\end{array}$} & \multirow[b]{2}{*}{$\begin{array}{l}\text { Labora- } \\
\text { torios }{ }^{\ddagger}\end{array}$} & \multirow[b]{2}{*}{$\begin{array}{c}\text { Rayos } \\
x^{\S}\end{array}$} \\
\hline & Total & $\begin{array}{l}\text { Consulta } \\
\text { externa }\end{array}$ & Hospitales & Censables & $\begin{array}{c}\text { No } \\
\text { censables }\end{array}$ & & & & & \\
\hline$N$ acional & 19253 & 18260 & 993 & 76865 & 59436 & 51213 & 2757 & 7061 & 1841 & 2115 \\
\hline Aguascalientes & 116 & 107 & 9 & 766 & 433 & 530 & 28 & 45 & 14 & 22 \\
\hline Baja California & 240 & 220 & 20 & 1643 & 778 & 1010 & 67 & 66 & 32 & 43 \\
\hline Baja California Sur & 148 & 130 & 18 & 576 & 403 & 383 & 28 & 51 & 23 & 22 \\
\hline Campeche & 276 & 256 & 20 & 673 & 595 & 673 & 28 & 55 & 23 & 22 \\
\hline Coahuila & 369 & 334 & 35 & 2216 & 1265 & 1270 & 80 & 74 & 57 & 65 \\
\hline Colima & 163 & 153 & 10 & 484 & 535 & 396 & 16 & 13 & 14 & 15 \\
\hline Chiapas & 1552 & 1510 & 42 & 1789 & 2357 & 2029 & 83 & 164 & 76 & 73 \\
\hline Chihuahua & 568 & 530 & 38 & 2417 & 1806 & 1443 & 86 & 170 & 69 & 66 \\
\hline Distrito Federal & 973 & 861 & 112 & 16753 & 6404 & 8079 & 592 & 132 & 300 & 466 \\
\hline Durango & 332 & 317 & 15 & 1145 & 1142 & 761 & 35 & 137 & 44 & 38 \\
\hline Guanajuato & 623 & 593 & 30 & 2528 & 2203 & 1676 & 87 & 399 & 48 & 76 \\
\hline Guerrero & 1043 & 1016 & 27 & 1594 & 2580 & 1803 & 71 & 689 & 73 & 61 \\
\hline Hidalgo & 747 & 722 & 25 & 1216 & 2148 & 1299 & 37 & 414 & 44 & 31 \\
\hline Jalisco & 1044 & 999 & 45 & 6155 & 4237 & 3077 & 219 & 555 & 97 & 118 \\
\hline México & 1208 & 1137 & 71 & 6970 & 4638 & 4301 & 183 & 410 & 138 & 170 \\
\hline Michoacán & 907 & 864 & 43 & 2134 & 2854 & 1757 & 89 & 260 & 68 & 59 \\
\hline Morelos & 276 & 264 & 12 & 844 & 915 & 748 & 33 & 141 & 19 & 21 \\
\hline $\bar{N}$ ayarit & 347 & 335 & 12 & 650 & 835 & 633 & 29 & 104 & 24 & 20 \\
\hline N uevo León & 555 & 528 & 27 & 3168 & 1716 & 1920 & 96 & 72 & 72 & 94 \\
\hline 0 axaca & 1258 & 1218 & 40 & 1803 & 2474 & 1943 & 65 & 497 & 54 & 49 \\
\hline Puebla & 960 & 911 & 49 & 3270 & 3091 & 2167 & 102 & 427 & 62 & 78 \\
\hline Q uerétaro & 301 & 293 & 8 & 757 & 965 & 649 & 27 & 155 & 19 & 23 \\
\hline Q uintana Roo & 211 & 196 & 15 & 556 & 489 & 543 & 28 & 112 & 33 & 21 \\
\hline San Luis Potosí & 552 & 532 & 20 & 1386 & 1300 & 1057 & 46 & 145 & 34 & 33 \\
\hline Sinaloa & 463 & 432 & 31 & 1936 & 1639 & 1220 & 86 & 207 & 46 & 56 \\
\hline Sonora & 369 & 329 & 40 & 2224 & 1621 & 1236 & 84 & 210 & 53 & 64 \\
\hline Tabasco & 611 & 586 & 25 & 1427 & 1617 & 1354 & 61 & 310 & 60 & 41 \\
\hline Tamaulipas & 508 & 472 & 36 & 2643 & 1686 & 1495 & 90 & 138 & 56 & 65 \\
\hline Tlaxcala & 198 & 189 & 9 & 553 & 754 & 474 & 21 & 154 & 16 & 13 \\
\hline $\begin{array}{l}\text { Veracruz } \\
\text {. }\end{array}$ & 1592 & 1515 & 77 & 4341 & 3907 & 3727 & 182 & 512 & 108 & 126 \\
\hline Yucatán & 335 & 317 & 18 & 1486 & 980 & 842 & 44 & 101 & 32 & 37 \\
\hline Zacatecas & 408 & 394 & 14 & 762 & 1069 & 718 & 34 & 142 & 33 & 27 \\
\hline
\end{tabular}

* No incluye sector privado

₹ Se refiere a laboratorios de análisis clínicos

$\S$ Se refiere a gabinetes de radiología

Fuente: Secretaría de Salud. Dirección General de Información y Evaluación del Desempeño. Boletín de Información Estadística N úmero 21,2001. México, 2002 


\section{Cuadro II \\ Principales Recursos físicos Y materiales por institución de salud. Sistema Nacional de Salud. México, 2001*}

\begin{tabular}{|c|c|c|c|c|c|c|c|c|c|c|}
\hline \multirow[b]{2}{*}{ Institución } & \multicolumn{3}{|c|}{ Unidades de salud } & \multicolumn{2}{|c|}{ Camas } & \multirow[b]{2}{*}{$\begin{array}{l}\text { Consul- } \\
\text { torios }\end{array}$} & \multirow[b]{2}{*}{$\begin{array}{l}\text { Quiró- } \\
\text { fanos }\end{array}$} & \multirow[b]{2}{*}{$\begin{array}{l}\text { Salas de } \\
\text { expulsión }\end{array}$} & \multirow[b]{2}{*}{$\begin{array}{l}\text { Labora } \\
\text { torios }^{\ddagger}\end{array}$} & \multirow[b]{2}{*}{$\begin{array}{c}\text { Rayos } \\
X^{\S}\end{array}$} \\
\hline & Total & $\begin{array}{l}\text { Consulta } \\
\text { externa }\end{array}$ & $\begin{array}{l}\text { Hospi- } \\
\text { tales }\end{array}$ & $\begin{array}{l}\text { Censa- } \\
\text { bles }\end{array}$ & $\begin{array}{c}\text { No } \\
\text { censables }\end{array}$ & & & & & \\
\hline Nacional & 19253 & 18260 & 993 & 76865 & 59436 & 51213 & 2757 & 7061 & 1841 & 2115 \\
\hline Población no derechohabiente & 15448 & 14942 & 506 & 34327 & 39951 & 28549 & 1234 & 6292 & 1019 & 790 \\
\hline Secretaría de Salud\# & 11835 & 11402 & 433 & 31914 & 32589 & 24256 & 1153 & 6216 & 945 & 712 \\
\hline Instituto Mexicano del Seguro Social-Solidaridad & 3609 & 3540 & 69 & 1999 & 7218 & 4193 & 69 & 69 & 69 & 69 \\
\hline Universitarios & 4 & 0 & 4 & 414 & 144 & 100 & 12 & 7 & 5 & 9 \\
\hline Población derechohabiente & 3805 & 3318 & 487 & 42538 & 19485 & 22664 & 1523 & 769 & 822 & 1325 \\
\hline Instituto Mexicano del Seguro Social & 1769 & 1510 & 259 & 28951 & 14483 & 13249 & 989 & 467 & 504 & 902 \\
\hline \multicolumn{11}{|l|}{ Instituto de Seguridad y Servicios Sociales } \\
\hline de los Trabajadores del Estado & 1247 & 1145 & 102 & 6730 & 2895 & 5397 & 288 & 165 & 202 & 270 \\
\hline Petróleos Mexicanos & 215 & 192 & 23 & 980 & 465 & 1538 & 52 & 28 & 22 & 32 \\
\hline Secretaría de la D efensa N acional & 341 & 299 & 42 & 4136 & 714 & 1273 & 93 & 52 & 45 & 53 \\
\hline Secretaría de Marina & 137 & 105 & 32 & 730 & 342 & 414 & 42 & 27 & 29 & 29 \\
\hline Estatales $^{\S}$ & 96 & 67 & 29 & 1011 & 586 & 793 & 59 & 30 & 20 & 39 \\
\hline \multicolumn{11}{|c|}{$\begin{array}{l}\text { * No incluye sector privado } \\
\text { ₹ Se refiere a laboratorios de análisis clínicos } \\
\text { § Se refiere a gabinetes de radiología } \\
\text { \# Incluye información de los Institutos N acionales de Salud } \\
\text { \& Incluye información del Sistema de Transporte Colectivo-Metro (STC-Metro) }\end{array}$} \\
\hline
\end{tabular}




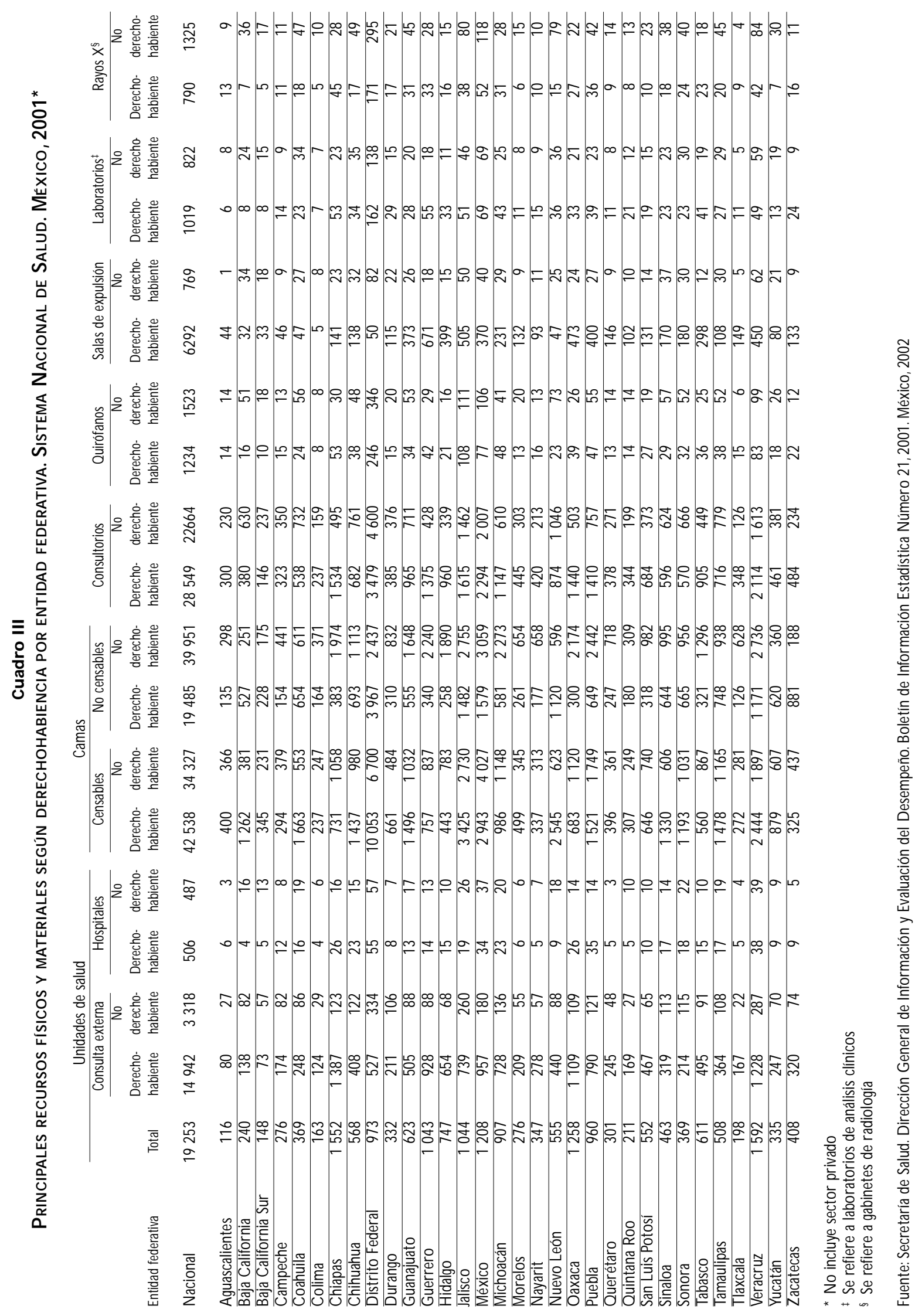




\section{Cuadro IV \\ Principales recursos humanos por entidad federativa. Sistema Nacional de Salud. México, 2001*}

\begin{tabular}{|c|c|c|c|c|c|c|c|c|c|c|}
\hline \multirow[b]{2}{*}{ Entidad federativa } & \multirow[b]{2}{*}{$\begin{array}{l}\text { Total de } \\
\text { personal }\end{array}$} & \multicolumn{5}{|c|}{ Médicos en contacto } & \multicolumn{4}{|c|}{ Paramédicos } \\
\hline & & $\begin{array}{l}\text { Total de } \\
\text { Médicos }\end{array}$ & $\begin{array}{l}\text { Gene- } \\
\text { rales }\end{array}$ & $\begin{array}{l}\text { Especia- } \\
\text { listas }\end{array}$ & $\begin{array}{l}\text { En for- } \\
\text { mación }{ }^{\ddagger}\end{array}$ & $\begin{array}{l}\text { Odon- } \\
\text { tólogos }\end{array}$ & $\begin{array}{l}\text { Médicos en } \\
\text { otras labores }\end{array}$ & $\begin{array}{l}\text { Enfer- } \\
\text { meras }\end{array}$ & $\begin{array}{l}\text { Otros para- } \\
\text { médicos }\end{array}$ & $\begin{array}{c}\text { Otro } \\
\text { personal\$ }\end{array}$ \\
\hline$N$ acional & 583430 & 141386 & 36808 & 44631 & 30122 & 8920 & 20905 & 189900 & 36389 & 215755 \\
\hline
\end{tabular}

\begin{tabular}{|c|c|c|c|c|c|c|c|c|c|c|}
\hline A guascalientes & 6885 & 1694 & 417 & 688 & 253 & 96 & 240 & 2269 & 448 & 2474 \\
\hline Baja California & 13898 & 3234 & 912 & 1244 & 357 & 204 & 517 & 4561 & 1118 & 4985 \\
\hline Baja California Sur & 4323 & 1098 & 309 & 387 & 156 & 64 & 182 & 1381 & 255 & 1589 \\
\hline Campeche & 4869 & 1316 & 479 & 397 & 224 & 80 & 136 & 1575 & 246 & 1732 \\
\hline Coahuila & 16387 & 3707 & 999 & 1264 & 488 & 195 & 761 & 5529 & 1261 & 5890 \\
\hline Colima & 3880 & 1050 & 245 & 391 & 213 & 51 & 150 & 1309 & 250 & 1271 \\
\hline Chiapas & 14782 & 3959 & 1263 & 1115 & 950 & 304 & 327 & 4887 & 833 & 5103 \\
\hline Chihuahua & 16928 & 3710 & 1195 & 1180 & 491 & 207 & 637 & 5593 & 1218 & 6407 \\
\hline Distrito Federal & 133699 & 29301 & 4664 & 10776 & 7016 & 1789 & 5056 & 41305 & 8510 & 54583 \\
\hline Durango & 11869 & 2879 & 730 & 1081 & 579 & 154 & 335 & 3874 & 759 & 4357 \\
\hline Guanajuato & 18127 & 4780 & 1460 & 1308 & 977 & 272 & 763 & 6105 & 1302 & 5940 \\
\hline Guerrero & 13296 & 3556 & 1426 & 938 & 638 & 225 & 329 & 4660 & 697 & 4383 \\
\hline Hidalgo & 10463 & 2801 & 776 & 705 & 738 & 187 & 395 & 3712 & 447 & 3503 \\
\hline Jalisco & 38049 & 9917 & 2192 & 2873 & 2798 & 499 & 1555 & 12911 & 2372 & 12849 \\
\hline México & 49536 & 11471 & 3319 & 3312 & 1936 & 930 & 1974 & 14619 & 3642 & 19804 \\
\hline Michoacán & 15539 & 4223 & 1055 & 1221 & 1124 & 347 & 476 & 5249 & 643 & 5424 \\
\hline Morelos & 7876 & 2013 & 509 & 771 & 316 & 151 & 266 & 2723 & 450 & 2690 \\
\hline $\mathrm{N}$ ayarit & 5554 & 1460 & 385 & 433 & 356 & 94 & 192 & 1957 & 282 & 1855 \\
\hline N uevo León & 24168 & 5367 & 1363 & 1586 & 1084 & 332 & 1002 & 8162 & 1771 & 8868 \\
\hline 0 axaca & 12950 & 3506 & 998 & 830 & 1153 & 220 & 305 & 4587 & 567 & 4290 \\
\hline Puebla & 19826 & 5354 & 1293 & 1374 & 1600 & 437 & 650 & 6696 & 889 & 6887 \\
\hline Q uerétaro & 7123 & 1844 & 524 & 566 & 377 & 120 & 257 & 2474 & 515 & 2290 \\
\hline Q uintana Roo & 5117 & 1346 & 496 & 420 & 155 & 82 & 193 & 1636 & 339 & 1796 \\
\hline San Luis Potosí & 10517 & 2619 & 720 & 696 & 704 & 189 & 310 & 3576 & 558 & 3764 \\
\hline Sinaloa & 15119 & 3489 & 1099 & 1108 & 552 & 183 & 547 & 5131 & 1035 & 5464 \\
\hline Sonora & 15750 & 3562 & 862 & 1279 & 677 & 152 & 592 & 5024 & 919 & 6245 \\
\hline Tabasco & 13338 & 3270 & 1189 & 892 & 590 & 292 & 307 & 4156 & 733 & 5179 \\
\hline Tamaulipas & 19321 & 4547 & 1282 & 1691 & 760 & 216 & 598 & 6332 & 1212 & 7230 \\
\hline Tlaxcala & 4937 & 1343 & 514 & 353 & 222 & 85 & 169 & 1613 & 284 & 1697 \\
\hline Veracruz & 33006 & 8780 & 3005 & 2490 & 1612 & 528 & 1145 & 10521 & 1941 & 11764 \\
\hline Yucatán & 10364 & 2586 & 678 & 803 & 606 & 133 & 366 & 3656 & 604 & 3518 \\
\hline Zacatecas & 5934 & 1604 & 450 & 459 & 420 & 101 & 174 & 2117 & 289 & 1924 \\
\hline
\end{tabular}

* No incluye sector privado

₹ Incluye residentes y pasantes

$\S$ Incluye auxiliares de diagnóstico y tratamiento, personal administrativo y otro personal

Fuente: Secretaría de Salud. Dirección General de Información y Evaluación del Desempeño. Boletín de Información Estadística Número 21, 2001. México, 2002 


\section{Cuadro V \\ Principales recursos humanos por institución. Sistema Nacional de Salud. MÉXICo, 2001*}

\begin{tabular}{|c|c|c|c|c|c|c|c|c|c|c|}
\hline & & & & M édicos en & contacto & & & Paran & médicos & \\
\hline Institución & $\begin{array}{l}\text { Total de } \\
\text { personal }\end{array}$ & $\begin{array}{l}\text { Total de } \\
\text { Médicos }\end{array}$ & $\begin{array}{l}\text { Gene- } \\
\text { rales }\end{array}$ & $\begin{array}{l}\text { Especia- } \\
\text { listas }\end{array}$ & $\begin{array}{l}\text { En for- } \\
\text { mación }\end{array}$ & $\begin{array}{l}\text { Odon- } \\
\text { tólogos }\end{array}$ & $\begin{array}{l}\text { Médicos en } \\
\text { otras labores }\end{array}$ & $\begin{array}{l}\text { Enfer- } \\
\text { meras }\end{array}$ & $\begin{array}{l}\text { Otros para- } \\
\text { médicos }\end{array}$ & $\begin{array}{c}\text { Otro } \\
\text { personal| }\end{array}$ \\
\hline Nacional & 583430 & 141386 & 36808 & 44631 & 30122 & 8920 & 20905 & 189900 & 36389 & 215755 \\
\hline Población no derechohabiente & 231100 & 62331 & 15501 & 16918 & 19615 & 5316 & 4981 & 81830 & 4984 & 81955 \\
\hline Secretaría de Salud\# & 211636 & 56113 & 15062 & 15824 & 15396 & 5161 & 4670 & 74475 & 4656 & 76392 \\
\hline Instituto Mexicano del Seguro Social-Solidaridad & 17500 & 5541 & 336 & 783 & 4026 & 137 & 259 & 6847 & 327 & 4785 \\
\hline Universitarios & 1964 & 677 & 103 & 311 & 193 & 18 & 52 & 508 & 1 & 778 \\
\hline Población derechohabiente & 352330 & 79055 & 21307 & 27713 & 10507 & 3604 & 15924 & 108070 & 31405 & 133800 \\
\hline Instituto Mexicano del Seguro Social & 258392 & 55842 & 15278 & 16767 & 7243 & 1817 & 14737 & 79715 & 24109 & 98726 \\
\hline Instituto de Seguridad y Servicios Sociales & & & & & & & & & & \\
\hline de losTrabajadores del Estado & 60204 & 15574 & 3728 & 7741 & 2572 & 765 & 768 & 19899 & 1872 & 22859 \\
\hline Petróleos Mexicanos & 10768 & 2392 & 900 & 1247 & 0 & 121 & 124 & 2673 & 1022 & 4681 \\
\hline Secretaría de la D efensa $\mathrm{N}$ acional & 10688 & 1859 & 468 & 516 & 174 & 618 & 83 & 2458 & 3969 & 2402 \\
\hline Secretaría de Marina & 3857 & 978 & 248 & 349 & 169 & 102 & 110 & 1102 & 182 & 1595 \\
\hline Estatales $^{\S}$ & 8421 & 2410 & 685 & 1093 & 349 & 181 & 102 & 2223 & 251 & 3537 \\
\hline $\begin{array}{l}\text { * No incluye sector privado } \\
\text { ₹ Incluye residentes y pasantes } \\
\S \text { Incluye auxiliares de diagnóstico y tratamiento, } \\
\text { \# Incluye información de los Institutos N acionales } \\
\text { \& Incluye información del Sistema de Transporte }\end{array}$ & $\begin{array}{l}\text { assonal ad } \\
\text { le Salud } \\
\text { lectivo-M }\end{array}$ & $\begin{array}{l}\text { ninistrativo } \\
\text { tro (STC- }\end{array}$ & $\begin{array}{l}\text { y otro } \\
\text { Metro) }\end{array}$ & ersonal & & & & & & \\
\hline
\end{tabular}




\section{Cuadro VI \\ Principales recursos humanos según tipo de derechohabiencia por entidad federativa. Sistema Nacional de Salud. México, 2001 ${ }^{\ddagger}$}

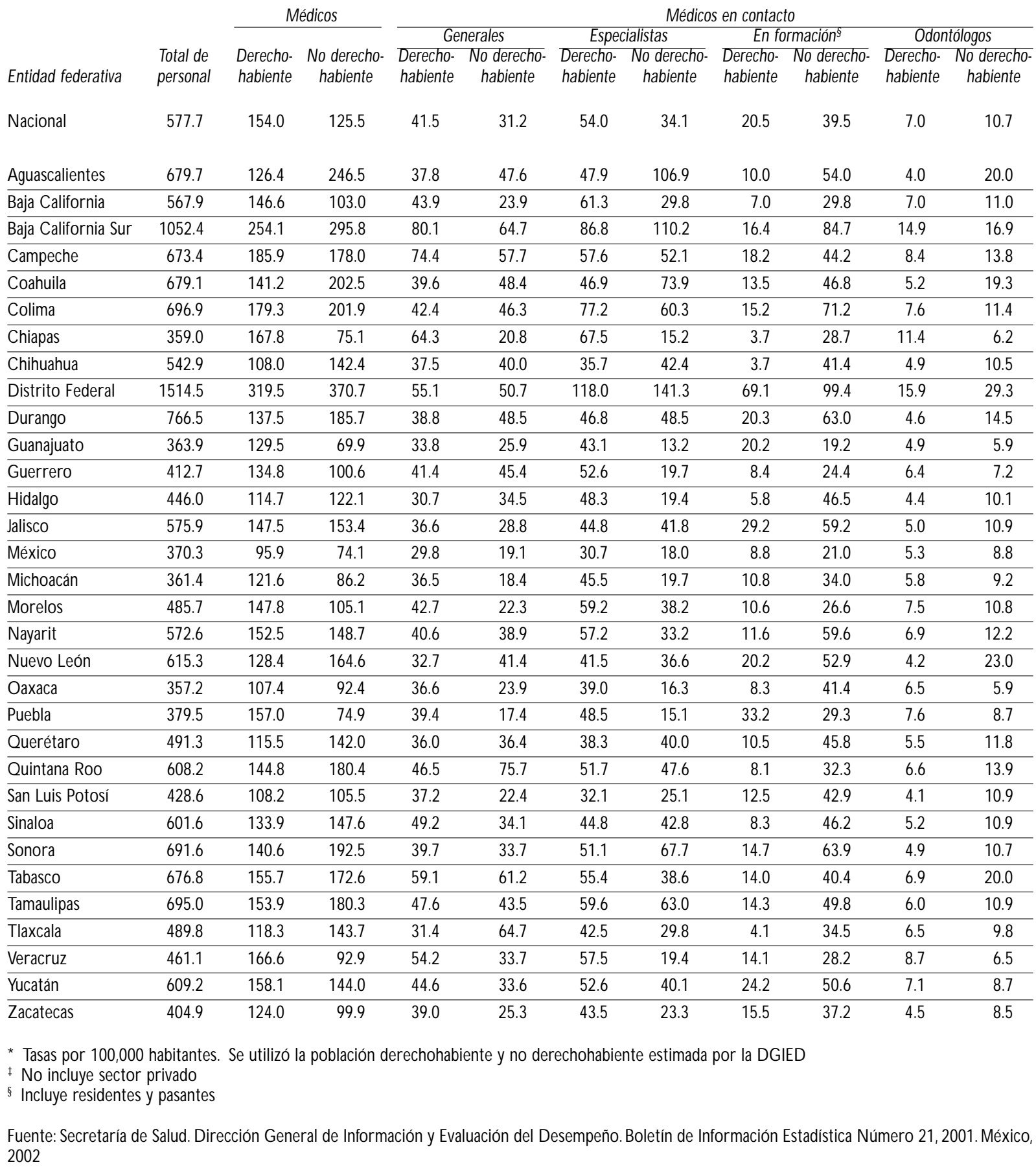




\section{Cuadro VII \\ Principales recursos humanos según tipo de derechohabiencia por entidad federativa.* Sistema Nacional de Salud. México, 2001}

\begin{tabular}{|c|c|c|c|c|c|c|c|c|}
\hline \multirow[b]{2}{*}{ Entidad federativa } & \multicolumn{2}{|c|}{ Médicos en otras labores } & \multicolumn{2}{|c|}{ Enfermeras } & \multicolumn{2}{|c|}{ Paramédicos } & \multicolumn{2}{|c|}{ Otro personal\$ } \\
\hline & $\begin{array}{l}\text { Derecho- } \\
\text { habiente }\end{array}$ & $\begin{array}{l}\text { No derecho- } \\
\text { habiente }\end{array}$ & $\begin{array}{l}\text { Derecho- } \\
\text { habiente }\end{array}$ & $\begin{array}{l}\text { No derecho- } \\
\text { habiente }\end{array}$ & $\begin{array}{l}\text { Derecho- } \\
\text { habiente }\end{array}$ & $\begin{array}{l}\text { No derecho- } \\
\text { habiente }\end{array}$ & $\begin{array}{l}\text { Derecho- } \\
\text { habiente }\end{array}$ & $\begin{array}{c}\text { No derecho- } \\
\text { habiente }\end{array}$ \\
\hline$N$ acional & 31.0 & 10.0 & 210.6 & 164.7 & 61.2 & 10.0 & 260.7 & 165.0 \\
\hline Aguascalientes & 26.6 & 18.0 & 169.9 & 329.0 & 56.5 & 20.3 & 203.9 & 322.6 \\
\hline Baja California & 27.3 & 8.6 & 196.5 & 165.9 & 64.7 & 7.4 & 237.8 & 135.1 \\
\hline Baja California Sur & 55.9 & 19.3 & 304.0 & 406.0 & 74.0 & 36.2 & 346.0 & 475.3 \\
\hline Campeche & 27.2 & 10.1 & 200.5 & 235.8 & 55.7 & 11.5 & 258.9 & 219.4 \\
\hline Coahuila & 35.9 & 14.2 & 215.9 & 281.2 & 61.0 & 17.8 & 246.4 & 234.7 \\
\hline Colima & 36.9 & 12.7 & 228.7 & 244.3 & 67.7 & 12.2 & 255.5 & 189.2 \\
\hline Chiapas & 20.8 & 4.2 & 175.8 & 101.9 & 68.3 & 6.1 & 254.4 & 85.6 \\
\hline Chihuahua & 26.2 & 8.0 & 167.7 & 204.2 & 53.0 & 9.2 & 216.3 & 182.3 \\
\hline Distrito Federal & 61.3 & 50.0 & 461.1 & 505.6 & 121.4 & 55.5 & 552.3 & 784.4 \\
\hline Durango & 27.0 & 11.3 & 178.2 & 237.7 & 50.6 & 12.7 & 214.0 & 188.3 \\
\hline Guanajuato & 27.5 & 5.8 & 172.1 & 84.1 & 54.7 & 4.0 & 198.5 & 57.7 \\
\hline Guerrero & 26.0 & 3.9 & 201.1 & 122.0 & 62.7 & 5.2 & 232.7 & 97.3 \\
\hline Hidalgo & 25.5 & 11.8 & 154.2 & 160.6 & 39.2 & 7.3 & 191.7 & 124.6 \\
\hline Jalisco & 32.0 & 12.7 & 199.7 & 189.9 & 53.3 & 13.6 & 220.0 & 161.6 \\
\hline México & 21.3 & 7.2 & 114.8 & 103.0 & 47.0 & 4.5 & 157.1 & 137.6 \\
\hline Michoacán & 23.0 & 5.0 & 163.7 & 100.8 & 36.2 & 4.1 & 202.8 & 87.0 \\
\hline Morelos & 27.8 & 7.2 & 201.6 & 140.8 & 53.1 & 7.3 & 258.6 & 91.3 \\
\hline$N$ ayarit & 36.2 & 4.7 & 216.1 & 188.6 & 55.4 & 4.9 & 288.6 & 102.1 \\
\hline N uevo León & 29.8 & 10.8 & 206.0 & 213.8 & 55.9 & 8.4 & 239.2 & 180.0 \\
\hline 0 axaca & 17.0 & 4.9 & 145.7 & 118.7 & 43.6 & 4.3 & 196.1 & 86.7 \\
\hline Puebla & 28.3 & 4.4 & 202.3 & 90.7 & 45.3 & 2.7 & 261.2 & 66.4 \\
\hline Q uerétaro & 25.3 & 8.2 & 157.1 & 188.0 & 55.3 & 10.3 & 181.2 & 128.4 \\
\hline Q uintana Roo & 31.9 & 10.9 & 183.8 & 208.8 & 65.3 & 6.7 & 252.7 & 160.9 \\
\hline San Luis Potosí & 22.2 & 4.2 & 148.2 & 143.6 & 41.3 & 6.4 & 181.4 & 128.8 \\
\hline Sinaloa & 26.3 & 13.7 & 197.4 & 216.1 & 59.2 & 9.4 & 228.5 & 197.8 \\
\hline Sonora & 30.1 & 16.6 & 187.4 & 296.3 & 49.7 & 19.1 & 257.1 & 313.1 \\
\hline Tabasco & 20.4 & 12.4 & 191.7 & 223.5 & 65.0 & 19.0 & 337.2 & 214.0 \\
\hline Tamaulipas & 26.4 & 13.2 & 202.4 & 271.4 & 60.2 & 15.1 & 257.7 & 264.1 \\
\hline Tlaxcala & 33.8 & 4.9 & 166.1 & 155.8 & 58.2 & 7.2 & 224.0 & 129.5 \\
\hline Veracruz & 32.1 & 5.1 & 213.1 & 102.1 & 60.6 & 4.4 & 290.0 & 79.1 \\
\hline Yucatán & 29.6 & 10.9 & 214.0 & 216.1 & 55.7 & 9.0 & 246.9 & 154.0 \\
\hline Zacatecas & 21.4 & 5.6 & 158.0 & 135.6 & 43.4 & 4.3 & 186.5 & 95.2 \\
\hline
\end{tabular}

* Tasas por 100,000 habitantes. Se utilizó la población derechohabiente y no derechohabiente estimada por la D GIED

₹ $\mathrm{No}$ incluye sector privado

$\S$ Incluye auxiliares de diagnóstico y tratamiento, personal administrativo y otro personal

Fuente: Secretaría de Salud. Dirección General de Información y Evaluación del Desempeño. Boletín de Información Estadística N úmero 21, 2001. México, 2002 


\section{Cuadro VIII}

Principales indicadores sobre recursos POR ENTIDAD FEDERATIVA. Sistema Nacional de Salud. México, 2001*

$\begin{array}{ccccc} & \text { Médicos por } & \text { Enfermeras } & \text { Consultorios } & \text { Camas por } \\ 100000 & \text { por } & \text { por } 100000 & 100000 \\ \text { Entidad federativa } & \text { habitantes }^{\ddagger} & \text { médico } & \text { habitantes } & \text { habitantes }\end{array}$

$\begin{array}{lllll}\text { N acional } & 119.3 & 1.6 & 50.7 & 76.1\end{array}$

$\begin{array}{lllll}\text { A guascalientes } & 143.5 & 1.6 & 46.0 & 75.6\end{array}$

\begin{tabular}{llllr}
\hline Baja California & 111.0 & 1.7 & 38.3 & 67.1 \\
\hline Baja California Sur & 223.0 & 1.5 & 91.3 & 140.2 \\
\hline Campeche & 163.2 & 1.3 & 72.3 & 93.1 \\
\hline Coahuila & 122.1 & 1.9 & 57.3 & 91.8 \\
\hline Colima & 161.7 & 1.5 & 73.6 & 86.9
\end{tabular}

\begin{tabular}{lrrrr}
\hline Colima & 161.7 & 1.5 & 73.6 & 86.9 \\
\hline Chiapas & 88.2 & 1.3 & 49.8 & 43.4 \\
\hline Chihuahua & 98.6 & 1.8 & 44.4 & 77.5
\end{tabular}

\begin{tabular}{lllll}
\hline Distrito Federal & 279.6 & 1.7 & 87.1 & 189.8 \\
\hline
\end{tabular}

\begin{tabular}{llllr}
\hline Durango & 136.1 & 1.5 & 57.3 & 73.9
\end{tabular}

\begin{tabular}{lllll}
\hline Guanajuato & 80.6 & 1.5 & 31.7 & 50.8 \\
\hline Guerrero
\end{tabular}

\begin{tabular}{lllll}
\hline Guerrero & 100.2 & 1.4 & 54.4 & 49.5 \\
\hline
\end{tabular}

\begin{tabular}{lllll}
\hline Hidalgo & 102.6 & 1.5 & 50.9 & 51.8 \\
\hline Jalisco & 126.6 & 1.5 & 48.0 & 93.2
\end{tabular}

\begin{tabular}{lllll}
\hline México & 71.0 & 1.5 & 35.3 & 52.1
\end{tabular}

\begin{tabular}{lllll}
\hline Michoacán & 87.1 & 1.4 & 41.9 & 49.6
\end{tabular}

\begin{tabular}{lllll}
\hline Morelos & 107.7 & 1.6 & 43.7 & 52.0 \\
\hline
\end{tabular}

\begin{tabular}{lrrrr}
\hline N ayarit & 130.7 & 1.5 & 62.9 & 67.0 \\
\hline Nuevo León & 111.1 & 1.9 & 57.6 & 80.7 \\
\hline O axaca & 88.3 & 1.4 & 48.3 & 49.7 \\
\hline Puebla & 90.0 & 1.4 & 40.8 & 62.6 \\
\hline Q uerétaro & 109.5 & 1.6 & 44.3 & 52.2 \\
\hline Q uintana Roo & 137.1 & 1.4 & 56.6 & 66.1 \\
\hline San Luis Potosí & 94.1 & 1.5 & 40.7 & 56.5 \\
\hline Sinaloa & 117.1 & 1.7 & 50.7 & 77.0 \\
\hline Sonora & 130.4 & 1.7 & 58.4 & 97.7 \\
\hline Tabasco & 150.3 & 1.4 & 64.2 & 72.4 \\
\hline Tamaulipas & 142.1 & 1.6 & 54.1 & 95.1 \\
\hline Tlaxcala & 116.5 & 1.4 & 44.0 & 54.9 \\
\hline Veracruz & 106.7 & 1.4 & 49.2 & 60.6 \\
\hline Yucatán & 130.5 & 1.6 & 46.8 & 87.4 \\
\hline Zacatecas & 97.6 & 1.5 & 48.5 & 52.0
\end{tabular}

* No incluye sector privado

₹ Se refiere a médicos en contacto con el paciente

§ Para el cálculo de los indicadores se utilizó la población estimada por el Consejo $\mathrm{N}$ acional de Población

Fuente: Secretaría de Salud. Dirección General de Información y Evaluación del Desempeño. Boletín de Información Estadística N 0. 21, 2001. México, 2002

\section{Cuadro IX \\ Principales indicadores sobre recursos POR INSTITUCIÓN DE SALUD. \\ Sistema Nacional de Salud. México, 2001* \\ Institución

$\begin{array}{cccc}\text { Camas } & \text { Médicos } & \text { Enfer- } & \text { Consulto- } \\ \text { por } & \text { por } & \text { meras } & \text { rios por } \\ 100000 & 100000 & \text { por } & 100000\end{array}$ $\begin{array}{cccc}100000 & 100000 \quad \text { por } & 100000 \\ \text { habitantes habitantes } & \text { médico habitantes }\end{array}$}

N acional $\begin{array}{llll}76.1 & 119.3 & 1.6 & 50.7\end{array}$

Población no derechohabiente

$69.1 \quad 115.5 \quad 1.3 \quad 57.5$

Secretaría de Salud ${ }^{\S}$ $\begin{array}{llll}72.3 & 116.6 & 1.4 & 55.0\end{array}$

Instituto Mexicano del Seguro

Social-Solidaridad

0 tras $^{\#}$

\begin{tabular}{rrrr}
18.7 & 49.3 & 1.3 & 39.2 \\
\hline 0.9 & 1.4 & 0.8 & 0.2
\end{tabular}

Población derechohabiente

$\begin{array}{llll}82.9 & 123.0 & 1.7 & 44.2\end{array}$

$\begin{array}{lllll}\text { Instituto Mexicano del Seguro Social } & 63.1 & 89.6 & 1.9 & 28.9\end{array}$ Instituto de Seguridad y Servicios

$\begin{array}{lllll}\text { Sociales de los Trabajadores del Estado } & 65.7 & 144.6 & 1.3 & 52.7\end{array}$

\begin{tabular}{lllll}
\hline Petróleos Mexicanos & 147.4 & 341.1 & 1.2 & 231.3 \\
\hline
\end{tabular}

\begin{tabular}{llllll}
\hline Secretaría de la D efensa N acional & 809.7 & 347.7 & 1.4 & 249.2 \\
\hline
\end{tabular}

\begin{tabular}{llllll}
\hline Secretaría de Marina & 396.1 & 439.5 & 1.4 & 224.6 \\
\hline
\end{tabular}

Estatales ${ }^{\&}$

$\begin{array}{llll}80.0 & 182.6 & 1.0 & 62.7\end{array}$

* No incluye sector privado

₹ Se refiere a médicos en contacto con el paciente

$\S$ Incluye información de los Institutos $N$ acionales de Salud

* Cifras estimadas

\& Incluye información del STC-Metro

* Para el cálculo de los indicadores se utilizó la población estimada por el Consejo N acional de Población; en el caso de las instituciones, las poblaciones de responsabilidad (legal o potencial)

Fuente:Secretaría de Salud. D irección G eneral de Información y Evaluación del D esempeño. Boletín de Información Estadística N 0.21, 2001. México, 2002 


\section{Cuadro X}

Camas Censables y no CENSABles de hospitales, 1997-2001

\begin{tabular}{|c|c|c|c|c|c|c|}
\hline & & 1997 & & & 2001 & \\
\hline & $\begin{array}{l}\text { Camas } \\
\text { Censables }\end{array}$ & $\begin{array}{c}\text { Camas } \\
\text { No censables }\end{array}$ & $\begin{array}{c}\text { Razón } \\
\text { CC/CNC }\end{array}$ & $\begin{array}{l}\text { Camas } \\
\text { Censables }\end{array}$ & $\begin{array}{c}\text { Camas } \\
\text { No censables }\end{array}$ & $\begin{array}{l}\text { Razón } \\
\text { CC/CNC }\end{array}$ \\
\hline$N$ acional & 71728 & 26131 & 2.7 & 73165 & 27177 & 2.7 \\
\hline A guascalientes & 796 & 282 & 2.8 & 756 & 256 & 3.0 \\
\hline Baja California & 1379 & 555 & 2.5 & 1529 & 597 & 2.6 \\
\hline Baja California Sur & 480 & 196 & 2.4 & 511 & 213 & 2.4 \\
\hline Campeche & 527 & 293 & 1.8 & 639 & 331 & 1.9 \\
\hline Coahuila & 2336 & 905 & 2.6 & 2124 & 787 & 2.7 \\
\hline Colima & 424 & 251 & 1.7 & 472 & 240 & 2.0 \\
\hline Chiapas & 1371 & 501 & 2.7 & 1493 & 593 & 2.5 \\
\hline Chihuahua & 2069 & 794 & 2.6 & 2322 & 909 & 2.6 \\
\hline Distrito Federal & 16518 & 5817 & 2.8 & 16456 & 5656 & 2.9 \\
\hline Durango & 1135 & 403 & 2.8 & 1189 & 480 & 2.5 \\
\hline Guanajuato & 2419 & 856 & 2.8 & 2317 & 751 & 3.1 \\
\hline Guerrero & 1255 & 553 & 2.3 & 1326 & 563 & 2.4 \\
\hline Hidalgo & 1203 & 334 & 3.6 & 1149 & 396 & 2.9 \\
\hline Jalisco & 5654 & 2048 & 2.8 & 5739 & 2156 & 2.7 \\
\hline México & 6464 & 2165 & 3.0 & 6745 & 2189 & 3.1 \\
\hline Michoacán & 1851 & 708 & 2.6 & 2038 & 901 & 2.3 \\
\hline Morelos & 777 & 393 & 2.0 & 833 & 379 & 2.2 \\
\hline $\mathrm{N}$ ayarit & 575 & 225 & 2.6 & 557 & 188 & 3.0 \\
\hline N uevo León & 3672 & 1426 & 2.6 & 3143 & 1328 & 2.4 \\
\hline 0 axaca & 1447 & 329 & 4.4 & 1708 & 474 & 3.6 \\
\hline Puebla & 2661 & 790 & 3.4 & 3106 & 934 & 3.3 \\
\hline Q uerétaro & 707 & 411 & 1.7 & 694 & 419 & 1.7 \\
\hline Q uintana Roo & 364 & 194 & 1.9 & 495 & 269 & 1.8 \\
\hline San Luis Potosí & 1409 & 407 & 3.5 & 1318 & 424 & 3.1 \\
\hline Sinaloa & 1787 & 734 & 2.4 & 1804 & 769 & 2.3 \\
\hline Sonora & 2302 & 854 & 2.7 & 2149 & 867 & 2.5 \\
\hline Tabasco & 1199 & 437 & 2.7 & 1334 & 552 & 2.4 \\
\hline Tamaulipas & 2545 & 1016 & 2.5 & 2592 & 1037 & 2.5 \\
\hline Tlaxcala & 404 & 166 & 2.4 & 486 & 270 & 1.8 \\
\hline Veracruz & 3939 & 1384 & 2.8 & 4064 & 1496 & 2.7 \\
\hline Yucatán & 1546 & 460 & 3.4 & 1447 & 477 & 3.0 \\
\hline Zacatecas & 513 & 244 & 2.1 & 630 & 276 & 2.3 \\
\hline
\end{tabular}

Fuente: Secretaría de Salud. Dirección General de Información y Evaluación del Desempeño. Boletín de Información Estadística N 0. 21, 2001. México, 2002 